(r)

\title{
Effect of operator experience and cementation strategies on the bond strength between fiber post and root dentin
}

César Dalmolin Bergoli, Julia Magalhães Costa Lima, Marco Antonio Bottino, Renata Marques de Melo \& Luiz Felipe Valandro

To cite this article: César Dalmolin Bergoli, Julia Magalhães Costa Lima, Marco Antonio Bottino, Renata Marques de Melo \& Luiz Felipe Valandro (2017) Effect of operator experience and cementation strategies on the bond strength between fiber post and root dentin, Journal of Adhesion Science and Technology, 31:1, 1-7, DOI: 10.1080/01694243.2016.1192012

To link to this article: https://doi.org/10.1080/01694243.2016.1192012

册 Published online: 24 Jun 2016.

Submit your article to this journal 주

Џ Article views: 50

View Crossmark data ¿

Citing articles: 1 View citing articles $\sqsubset \pi$ 


\title{
Effect of operator experience and cementation strategies on the bond strength between fiber post and root dentin
}

\author{
César Dalmolin Bergolia, Julia Magalhães Costa Lima ${ }^{b}{ }$, Marco Antonio Bottino ${ }^{b}$, \\ Renata Marques de Melo ${ }^{b}$ and Luiz Felipe Valandroc
}

aFaculty of Dentistry, Department of Restorative Dentistry, Federal University of Pelotas, Rio Grande do Sul, Brazil; 'bScience and Technology Institute, São Paulo State University (UNESP), Sao Paulo, Brazil; cFaculty of Dentistry, Department of Restorative Dentistry, Federal University of Santa Maria, Rio Grande do Sul, Brazil

\begin{abstract}
This study evaluates the influence of operator experience on the bond strength between fiber posts and root dentin using different cementation strategies. One hundred and twenty bovine teeth were selected, cleaned, disinfected, and sectioned at $15 \mathrm{~mm}$ of root length (apical-coronal). The root canals were prepared up to $12 \mathrm{~mm}$ and the apical portions were embedded with acrylic resin in plastic matrices. Thereafter, the teeth were randomly distributed to six operators (three with experience and three without experience). The operators performed cementation using two different cementation strategies: conventional (three-step etch-and-rinse adhesive; Scotchbond MultiPurpose and RelyX ARC) or simplified (one-step self-adhesive resin cement; RelyX U200). Following cementation, each specimen was sectioned to obtain three slices $(2 \mathrm{~mm})$. Slices were submitted to the push-out test and failure analysis. Mean bond strength of each group was evaluated by the Student's $t$-test $(a=0.05)$. Statistical analysis did not show any influence of operator experience or cementation strategy on the bond strength values. The principal mode of failure was adhesive between the resin cement and root dentin. Authors conclude that operators with different clinical experience could achieve similar values of bond strength between fiber posts and root dentin, independent of the cementation strategy (conventional or simplified).
\end{abstract}

\section{ARTICLE HISTORY}

Received 23 March 2016

Revised 10 May 2016

Accepted 11 May 2016

\section{KEYWORDS}

Bond strength; fiber post; resin cement; root dentin; push-out test

\section{Introduction}

The use of fiber posts has become widespread in dentistry, especially because they decrease the risk of root fracture and are more conservative than cast posts and cores.[1,2] The bond between fiber posts and root dentin is achieved through different adhesive cementation approaches, either using adhesive systems associated with resin cements (conventional) or by the use of self-adhesive resin cements (simplified). 
Clinical studies have demonstrated high rates of clinical success for fiber posts; however, failures related to decementation have also been reported.[1-3] Thus, studies comparing different adhesive approaches have continued to be performed in the search for the most reliable cementation strategy.

Some studies have demonstrated better bond strength between fiber posts and root dentin when a three-step total etch-and-rinse adhesive system is associated with a conventional resin cement. [4-7] However, this technique is very sensitive and its execution is more difficult than that of others, [8-10] especially because of the need to control the moisture inside the root canal. Consequently, the clinical experience of the operator appears to be a very important factor in ensuring a reliable union between the fiber post and root dentin.[10-12]

To decrease technique sensitivity and the clinical time expended during the cementation procedures, the self-adhesive resin cement was developed. As this does not entail any pre-treatment of the dentin/enamel surface, this material is a quick and easy alternative for the dentist. The simplicity of this cement is further advantageous in fiber post cementation, which is very difficult to control inside the root canal.

Some studies have compared the bond strength values between self-adhesive cements (simplified) and resin cements associated with the three-step etch-and-rinse adhesives, with no consensus on which one constitutes the better strategy.[4,5,13-15] However, these studies did not consider the influence of the operator's ability to perform a fiber post cementation on the bond strength values obtained by the different strategies. It could be that the high values of bond strength demonstrated by the conventional resin cements associated with the three-step etch-and-rinse adhesive were achieved by trained and experienced operators.

Therefore, this study aimed to evaluate, through the push-out test, the influence of operator experience on the bond strength between fiber posts and root dentin, using two different cementation strategies. The hypotheses tested were as follows: (1) operator experience influences the bond strength between fiber posts and root dentin, independent of the cementation strategy; (2) cementation strategy does not influence the bond strength between fiber posts and root dentin, independent of operator experience.

\section{Materials and methods}

\subsection{Selection and specimen preparation}

Extracted bovine mandibular anterior teeth were selected, cleaned, disinfected, and stored in distilled water $\left(4^{\circ} \mathrm{C}\right)$. The coronal portions were sectioned, standardizing the root length to $15 \mathrm{~mm}$. Further, the root canal diameter was measured, serving as an inclusion criterion. Teeth were excluded from the study if the diameter of the root canal exceeded $2.2 \mathrm{~mm}$, which corresponds to the diameter of the fiber post used in the study (\#3, White Post DC; FGM, Joinville, Brazil). Thereafter, 120 specimens were selected $(N=120)$ for the study.

After the selection procedure, specimens were manually instrumented using NiTi endodontic instruments (\#40, ProTaper; Dentsply-Maillefer, Petrópolis, RJ, Brazil) under constant irrigation with $1 \%$ sodium hypochlorite to remove the remnants of pulp. Further, the root canals were mechanically prepared up to $12 \mathrm{~mm}$ length using the bur specific to the fiber post system (\#3, White Post DC; FGM). Finally, the root apices were covered with a resin composite (Opallis; FGM) and embedded with chemically cured acrylic resin (Dencrilay; Dencril, Caieiras, SP, Brazil) in a plastic matrix, in accordance with the methodology employed by Bergoli et al. [13]. 
Table 1. Experimental design of the study and cementation procedures.

\begin{tabular}{|c|c|c|c|c|}
\hline Operators & $\begin{array}{l}\text { Operators } \\
\text { experience }\end{array}$ & Cementation strategies & $\begin{array}{l}\text { Adhesive } \\
\text { procedures }\end{array}$ & $\begin{array}{l}\text { Groups } \\
(n=30)\end{array}$ \\
\hline \multirow[t]{2}{*}{$\begin{array}{l}\text { Operators1, } 2 \\
\text { and } 3\end{array}$} & $\begin{array}{l}\text { Without } \\
\text { experience }\end{array}$ & $\begin{array}{l}\text { Three step etch-and-rinse adhesive system* } \\
+ \text { conventional resin cement** }\end{array}$ & $a, b, c, d, e, f$ & G1 \\
\hline & & Self adhesive resin cement + & $e, f$ & G2 \\
\hline \multirow[t]{2}{*}{$\begin{array}{l}\text { Operators 3, } 4 \\
\text { and } 5\end{array}$} & $\begin{array}{l}\text { With } \\
\text { experience }\end{array}$ & $\begin{array}{l}\text { Three-step etch-and-rinse adhesive system* } \\
+ \text { conventional resin cement** }\end{array}$ & $a, b, c, d, e, f$ & G3 \\
\hline & & Self adhesive resin cement $†$ & $e, f$ & G4 \\
\hline
\end{tabular}

Note. Adhesive procedures: (a) etching the root canal with a $37 \%$ phosphoric acid during $15 \mathrm{~s}$; (b) cleaning the root canal with distilled water; (c) removal of water excess with endodontic absorbent paper points \#40; (d) application of primer, activator e catalyst of the adhesive system Scotch Bond Multi Purpose with microbrush (3M, ESPE); (e) manipulation and insertion of the resin cement with syringe Centrix (DFL, Rio de Janeiro, RJ, Brazil) using acudose point tips (DFL, Brasil); (f) remotion of the resin cement excess and photo activation for $40 \mathrm{~s}$ com LED (Radii Cal, SDI, Australia).

* Scotch Bond Multi Purpose (3M ESPE, MN, USA)

**RelyX ARC (3M ESPE)

tRelyX U100.

\subsection{Randomization process, operator selection, and cementation procedures}

Specimens were allocated in accordance with operator experience and cementation strategy (Table 1). Operator experience was classified as follows: operators who had never cemented any fiber post and/or never used the adhesive materials before were classified as 'without experience;' operators who had cemented at least 20 fiber posts and had already used the adhesive materials were classified as 'with experience.' All operators were postgraduate students.

All operators received written instructions regarding the cementation procedures, in accordance with the manufacturer's recommendations. Prior to cementation, all fiber posts were cleaned with $96 \%$ alcohol and silanized (ProSil; FGM).

After the fiber post cementation, a coronal reconstruction was performed with composite resin (Oppalis; FGM) using plastic matrices of standardized dimensions [16] and the specimens were immersed in distilled water for $24 \mathrm{~h}$ at $37^{\circ} \mathrm{C}$, before testing.

\subsection{The push-out test for bond strength}

The specimens were attached to a metal base screwed to a cutting machine (LabCut 1010; Extec Corp., Enfield, CT, USA) and sectioned perpendicular to the root axis. The first cervical slice $(1 \mathrm{~mm})$ was discarded and three additional slices $(2 \mathrm{~mm})$ were obtained. Immediately after the sectioning procedures, the disk specimens were positioned on a pushout testing device (metal rod with a central opening larger than the root canal diameter; $\varnothing=3 \mathrm{~mm}$ ). The most coronal face of the disk was placed downwards. A metal device for the push-out test $(\varnothing=0.85 \mathrm{~mm})$ induced a load apically on the post, without applying any pressure on the dentin or cement. The test was executed on a universal testing machine (DL 2000; EMIC, São José dos Pinhais, PR, Brazil) at $1 \mathrm{~mm} / \mathrm{min}$.

The bond strength was calculated using the formula $R=C / A$, where $C$ is the fracture load $(N)$ and $A$ is the bonded area $\left(\mathrm{mm}^{2}\right)$. To calculate the bonded area, the following formula was used: $A=\pi g \cdot\left(R_{1}+R_{2}\right)$, where $A=$ interfacial area, $\pi=3.14, g=$ height, $R_{1}=$ smaller base radius, and $R_{2}=$ larger base radius. To determine the height, the following formula was used: $g^{2}=h^{2}+\left[R_{2}-R_{1}\right]^{2}$, where $h=$ section height. $R_{1}$ and $R_{2}$ were obtained by measuring 
the internal diameters of the smaller and larger base using a digital caliper (Starrett 727; Starrett, Itu, SP, Brazil).

Specimens with cohesive fractures of the post or dentin were excluded from the study because they did not express the real push-out bond strength values between the fiber post and root dentin.

The push-out test for all specimens was performed by a single calibrated operator and was blinded for group conditions.

\subsection{Failure analysis}

All disk specimens were examined under a stereomicroscope (Discovery V-20; Zeiss, Germany) to categorize the mode of failure. The different modes of failure were as follows: Ades CD (adhesive failure between resin cement and dentin); Ades CP (adhesive failure between resin cement and fiber post); Coes $\mathrm{C}$ (cohesive failure of resin cement); Coes $\mathrm{P}$ (cohesive failure of fiber post); and Coes D (cohesive failure of dentin).

\subsection{Statistical analysis}

Initially, sample size calculation was performed assuming the following parameters: standard deviation of 3.5 MPa for the control group (based on Simonetti et al. 2006), statistically significant difference of $5 \mathrm{MPa}$ between the control and experimental groups, $90 \%$ power of the sample size, and a 5\% significance level $(\alpha=0.05)$. The sample size calculation was performed by a computer program available on the website www.lee.dante.br and the sample size was defined as 10 specimens $(n=10)$ in each group.

The mean bond strength values of all the groups were submitted to the Student's $t$-test to analyze the influence of operator experience and cementation strategy on the results $(\alpha=0.05)$. The coefficient of variation $(\mathrm{Cv})$ of each group was also calculated using the formula: $\mathrm{Cv}=100 \mathrm{~s} / x$, where $\mathrm{Cv}=$ variation coefficient, $s=$ standard deviation, and $x=$ mean bond strength.

\section{Results}

Student's $t$-test did not show any statistically significant difference between the groups (Table 2). The coefficient of variation showed higher variability of the bond strength values in groups without experience (Table 3).

Adhesive failures between resin cement were predominant (62\%), followed by adhesive failures between fiber posts and resin cement (30\%) and dentin cohesive failures (8\%). Figure 1 shows a stereomicroscope image of the failure patterns observed.

Table 2. Mean, standard deviation (MPa) and statistical differences after T-Student test $(a=0.05)$.

\begin{tabular}{|c|c|c|c|}
\hline \multirow[b]{2}{*}{ Operator's experience } & \multicolumn{3}{|c|}{ Cementation strategy } \\
\hline & Conventional* & & Simplified* \\
\hline Yes & $\begin{array}{c}16.08(5.5)^{\mathrm{A}, \mathrm{a}} \\
(p=0.953)\end{array}$ & $(p=0.053)$ & $\begin{array}{c}13.56(3.71)^{\mathrm{B}, \mathrm{a}} \\
(p=0.214)\end{array}$ \\
\hline No & $15.92(6.78)^{A, b}$ & $(p=0.510)$ & $14.91(4.81)^{\mathrm{B}, \mathrm{b}}$ \\
\hline
\end{tabular}

${ }^{*}$ Capital letters indicate comparison between columns. Lowercase letters indicate comparison between lines. Identical letters indicate similar statistically significant results. 
Table 3. Coeficient of variation of the groups (\%).

\begin{tabular}{lcc}
\hline & \multicolumn{2}{c}{ Cementation strategy } \\
\cline { 2 - 3 } Operator experience & Conventional (\%) & Simplified (\%) \\
\hline Yes & 34,2 & 27,3 \\
No & 42,5 & 32,2 \\
\hline
\end{tabular}

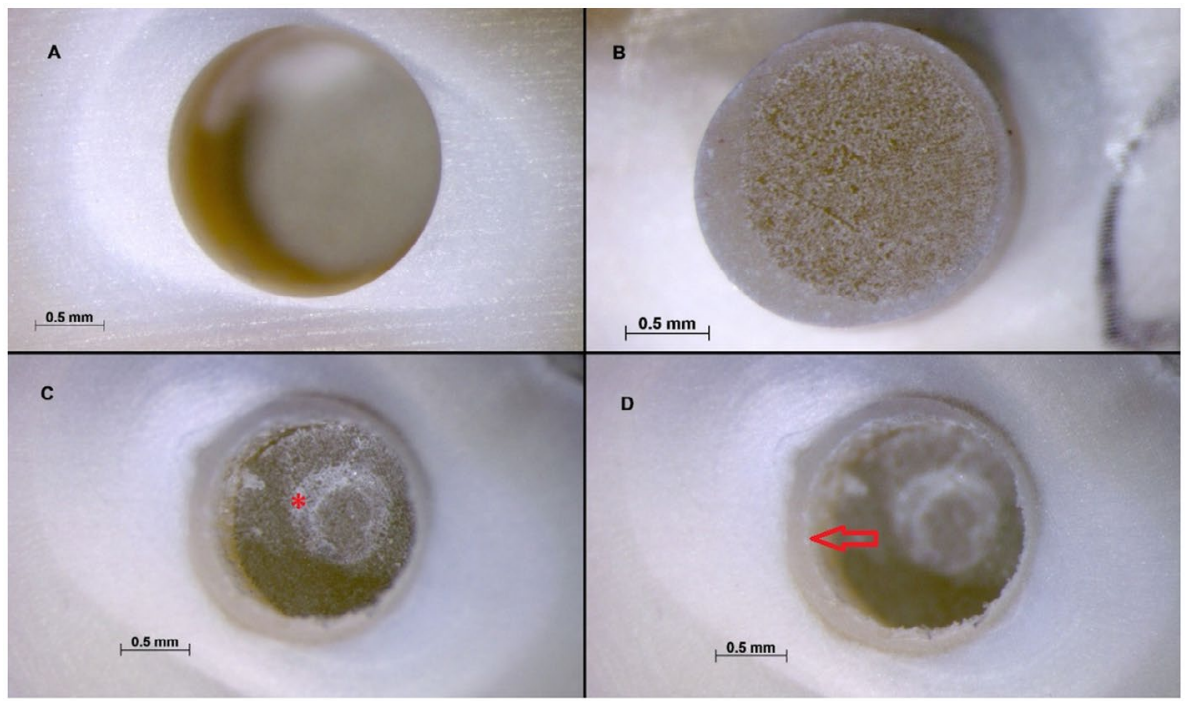

Figure 1. Figures $A$ and $B$ show an adhesive failure between resin cement and dentin; figures $C$ and $D$ show an adhesive failure between resin cement and fiber post. The asterisk indicates the fiber posts, while the arrow indicates the resin cement layer fixed in the dentin walls.

\section{Discussion}

Operator experience could be an important factor in the performance of restorative materials. [10-12,17] However, there are few studies evaluating the influence of operator experience on the bond strength between fiber posts and root dentin by varying the cementation strategy (conventional cementation vs simplified cementation).

The results of this study showed that the bond strength values between fiber posts and root dentin were not influenced by operator experience, thus rejecting the first hypothesis. This result disagrees with that of other studies which showed an influence of operator experience on adhesive procedures performed on coronal dentin,[10-12] with higher bond strength values observed in groups restored by operators with clinical experience. On the other hand, the current study evaluates the influence of operator experience during fiber post cementation, which presents a technique that is more sensitive, thus affecting the quality of bonding procedures in both operator groups. Furthermore, in the current study, the 'without experience' operators were postgraduate students with the scientific knowledge of the functional properties of the materials, which in accordance with Unlu et al. [10], is as important as the operator's clinical experience.

Although operator experience had no significant influence on the bond strength values (Table 2), significant differences in the coefficient of variation values (Table 3 ) were observed 
between groups with and without experience. The specimens cemented by operators with experience showed lower bond strength variation, which means there was a small chance of occurrence of an error during the cementation procedure.

Cementation strategy (conventional/simplified) did not influence bond strength between fiber posts and root dentin, independent of operator experience, thus accepting the second hypothesis of the study. This result is in accordance with that of other studies $[4,13,18]$ and could be related to the quality of both strategies. The total etch-and-rinse approach is considered a gold standard and its functionality is well established in the literature.[4-7] On the other hand, the high values of bond strength reached by self-adhesive resin cements could be related to the multi-functional phosphoric acid monomers, $[4,13,16]$ which produce a strong bond with the cement matrix during the polymerization process, resulting in suitable bond strength and dimensional stability of the cement, and promoting adhesion.[16] Further, at the beginning of the reaction, the cement has a very low $\mathrm{pH}$, which allows the hydrophilic properties of the material to provide good adaptation to the root walls. During the course of the reaction, acidic monomers interact with the filler components of the cement and the hydroxyapatite content in dentin, neutralizing the reaction and increasing the $\mathrm{pH}$.[19] The neutralization reaction allows the material to acquire hydrophobic properties and an alkaline character, resulting in low coefficients of expansion and solubility, which are essential for good long-term behavior.[16]

Identical to operator experience, differences in the coefficient of variation were observed for cementation strategy as well (Table 3). Independent of operator experience, it was possible to observe that the simplified strategy generated lower results for variability. It could be related to the fact that this strategy involves just one step, without necessitating any previous root canal treatment and allowing the execution of the technique more reliably with lesser chances of error.

The predominant mode of failure observed in this study was that of the adhesive between the resin cement and root dentin (Figure 1(A)). This type of failure is the same as that observed in clinical studies, [1-3] evidencing the difficulty to reach good patterns of union inside the root canal. The main factors that affect union with root dentin are the difficulty in controlling moisture inside the root canal, the high C-factor of the cavity,[20] and the decreased intensity of light transmission through the root.[21] Another important factor to note in the current study is that the bond strength evaluation was performed $24 \mathrm{~h}$ after the cementation procedure, without any aging protocol. Considering this, it may not be sensible to relate the outcomes of this study with the clinical outcomes of fiber posts. Further in vitro studies applying aging protocols and clinical studies verifying the influence of operator experience on the behavior of endodontically treated teeth restored with fiber posts would be interesting contributions to the subject.

Therefore, this study concludes that: (1) the operator experience and cementation strategy did not influence the values of bond strength between fiber posts and root dentin; (2) operators with clinical experience and the simplified cementation strategy are more reliable, with less chances of failure during the adhesion procedure.

\section{Funding}

This research work supported by the Sao Paulo Research Foundation (FAPESP; São Paulo, Brazil) [\#2011/09897-8]. 


\section{References}

[1] Ferrari M, VIchi A, Mannocci F, et al. Retrospective study of the clinical performance of fiber posts. Am. J. Dent. 2000;13:9B-13B.

[2] Malferrari S, Monaco C, Scotti R. Clinical evaluation of teeth restored with quartz fiberreinforced epoxy resin posts. Int. J. Prosthodont. 2003;16:39-44.

[3] Schmitter M, Rammelsberg P, Gabbert O, et al. Influence of clinical baseline findings on the survival of 2 post systems: a randomized clinical trial. Int. J. Prosthodont. 2007;20:173-8.

[4] Amaral M, Santini MF, Wandscher V, et al. An in vitro comparison of different cementation strategies on pull-out strength of a glass fiber post. Oper. Dent. 2009;34:443-451.

[5] Bitter K, Paris S, Pfuertner C, et al. Morphological and bond strength evaluation of different resin cements to root dentin. Eur. J. Oral Sci. 2009;117:326-333.

[6] Bonfante G, Kaizzer OB, Pegoraro LF, et al. Tensile bond strength of glass fiber posts luted with different cements. Braz. Oral Res. 2007;21:159-164.

[7] Mello RM, Galhano G, Barbosa SH, et al. Effect of type of adhesive system and tooth region on the bond strength to dentin. J. Adhes. Dent. 2008;10:127-33.

[8] Ferrari M, Tay FR. Technique sensitivity in bonding to vital, acid etched dentin. Oper. Dent. 2003;28:3-8.

[9] Sano H, Kanemura N, Burrow MF, et al. Effect of operator variability on dentin adhesion: students vs dentists. Dent. Mater. J. 1998;17:51-58.

[10] Unlu N, Gunal S, Ulker M, et al. Influence of operator experience on in vitro bond strength of dentin adhesives. J. Adhes. Dent. 2011;13:1-5.

[11] Adebayo OA, Burrow MF, Tyas MJ. Bond strength test: role of operator skill. Aust. Dent. J. 2008;53:145-150.

[12] Ueda M, Mine A, De Munck J, et al. The effect of clinical experience on dentine bonding effectiveness: students versus trained dentists. J. Oral Rehabil. 2010;37:653-657.

[13] Bergoli CD, Amaral M, Boaro LC, et al. Fiber post cementation strategies: effect of mechanical cycling on push-out bond strength and cement polymerization stress. J. Adhes. Dent. 2012;14:471-478.

[14] de Durâo Mauricio PJBT, González-López S, Aguilar-Mendoza JA, et al. Comparison of regional bond strength in root thirds among fiber-reinforced posts luted with different cements. J. Biomed. Mater. Res. B: Appl. Biomater. 2007;83B:364-372.

[15] Zicari F, Couthino E, De Munck J, et al. Bonding effectiveness and sealing ability of fiber-post bonding. Dent. Mater. 2008;24:967-977.

[16] Radovic I, Monticelli F, Goracci C, et al. Self-adhesive resin cements: a literature review. J. Adhes. Dent. 2008;10:251-258.

[17] Miyazaki M, Onose H, Moore BK. Effect of operator variability on dentin bond strength of two step bonding systems. Am. J. Dent. 2000;13:101-104.

[18] Gomes G, Gomes O, Reis A, et al. Effect of operator experience on the outcome of fiber post cementation with different resin cements. Oper. Dent. 2013;38:555-564.

[19] Saskalauskaite E, Tam LE, McComb D. Flexural strength, elastic modulus, and ph profile of self-etch resin luting cements. J. Prosthodont. 2008;17:262-268.

[20] Bouillaguet S, Troesch S, Wataha JC, et al. Microtensile bond strength between adhesive cements and root canal dentin. Dent. Mater. 2003;19:199-205.

[21] Morgan LFSA, Peixoto RTRC, Albuquerque RC, et al. Light transmission through a translucent fiber post. J. Endod. 2008;34:299-302. 